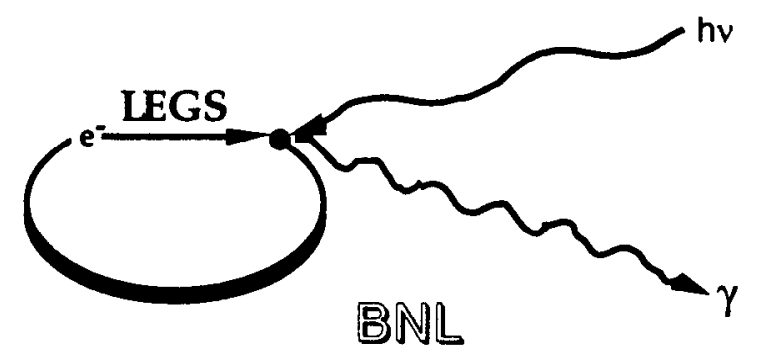

BNL- 66320

\title{
Production of high-power CW UV by Resonant Frequency Quadrupling of a Nd:YLF Laser
}

\begin{abstract}
Anthony J. Kuczewski ${ }^{a}$, Craig E. Thorn ${ }^{a}$, Giovanni Matone $^{\mathrm{b}}$, Gianfranco Giordano ${ }^{\mathrm{b}}$
\end{abstract}

a Physics Department, Brookhaven National Laboratory, Upton N.Y. 11973, USA

bINFN-Laboratori Natzionali di Frascati, Frascati, Italy

\section{Laser Electron Gamma Source}

Brookhaven National Laboratory

Brookhaven Science Associates

Under Contract \# DE-AC02-98CH10886 with the United States Department of Energy 


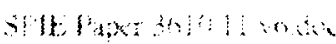

\title{
Production of high-power CW UV by resonant frequency quadrupling of a Nd:YLF laser
}

\author{
Anthony J. Kuczewski", Craig E. Thorn", Giovanni Matone", Gianfranco Giordano ${ }^{b}$ \\ " Department of Physics, Brookhaven National Lab, Upton, N.Y. 11973 \\ ' INFN-Laboratori Natzionali di Frascati, Frascati, Italy
}

\begin{abstract}
We have constructed a single ring to resonantly double an 18 watt Nd:YLF mode-locked laser and re-double the stored green to produce over 4 watts of power in the ultra-violet (UV). This laser is used to produce a beam of $470 \mathrm{MeV}$ gamma-rays by Compton backscattering the laser beam from $2.8 \mathrm{GeV}$ electrons stored in a synchrotron. Achieving high luminosity of the colliding beams requires very good mode quality and beam stability at the intersection point 22 meters from the laser. The ring consists of six mirrors, with two $25 \mathrm{~cm}$ radius of curvature mirrors enclosing each nonlinear crystal. The drive laser is a lamp-pumped Nd:YLF with a 50 ps bunch length at $76 \mathrm{MHz}$. A pointing stabilizer servo has been constructed as part of the infrared (IR) mode matching telescope. The IR to green conversion is accomplished in a $15 \mathrm{~mm}$ long non-critically phased matched LBO crystal located at a 40 micron waist, with an IR conversion efficiency of $70 \%$. A stable, nearly diffraction limited UV beam of up to 4.2 watts is generated in a BBO crystal in the green storage ring. The output power is relatively independent of the efficiency of the LBO and BBO crystals. This fact makes it possible to reduce the amount of non-TEM ${ }_{00}$ modes created by walk-off of the UV by using relatively thin BBO crystals. At present, however, the lower bound on the BBO thickness is limited by the loss of conversion efficiency at high power.
\end{abstract}

Keywords: resonant cavity, high power UV, second harmonic generation, frequency quadrupling, BBO, LBO

\section{INTRODUCTION}

The Laser-Electron-Gamma-Source (LEGS) facility ${ }^{1}$ at Brookhaven National Laboratory (BNL) produces a beam of high energy gamma rays by Compton backscattering a laser beam from the circulating electron beam in the National Synchrotron Light Source (NSLS) storage ring. The NSLS "x-ray" ring stores relativistic electrons of energy 2.584 or $2.800 \mathrm{GeV}$. The maximum gamma ray energy produced in this collision can be computed simply by doppler shifting the incoming laser photon (of energy $\varepsilon$ ) into the electron rest frame, Compton scattering at 180 degrees in the electron rest frame, and then doppler shifting the scattered photon back into the lab frame. The relativistic doppler shift is $2 \gamma$, where the Lorentz factor is $\gamma=$ $1+\mathrm{E}_{\mathrm{c}} / \mathrm{mc}^{2}$, for an electron kinetic energy of $\mathrm{E}_{\mathrm{c}}$ and mass of $\mathrm{m}$. The Compton scattering causes the electron at rest to recoil, reducing the photon energy by $1 /\left(1+4 \varepsilon \gamma / \mathrm{mc}^{2}\right)$. The resulting energy of the backscattered laser light is the product of these three factors:

$$
\frac{E_{\gamma}}{\varepsilon}=\frac{4 \gamma^{2}}{1+4 \gamma \varepsilon / \mathrm{c}^{2}}
$$

For a $2.8 \mathrm{GeV}$ electron beam $(\gamma=5480)$ and an ultraviolet photon the collision produces an energy gain of about $10^{8}$, converting the light into gamma rays of energy up to $500 \mathrm{MeV}$. The scattered photons are formed into a beam by the Lorentz contraction, which collapses the scattering distribution in the electron rest frame into a cone in the lab system of half angle $1 / \gamma$ (about 180 microradians). The polarization of the laser photon is preserved to a high degree in the collision, making it easy to produce highly polarized gamma ray beams. Thus this beam is an excellent tool for studying the structure, and in particular the spin structure, of the nucleon and its first excited state, the $\Delta$ resonance, located at $320 \mathrm{MeV}$ excitation energy with a width of $120 \mathrm{MeV}$. For examples of this research see ${ }^{2,3}$.

Most of the nuclear physics research at LEGS has been performed with a Coherent Corporation Innova Ar-Ion laser producing about 5 Watts at 333, 351, and $363 \mathrm{~nm}$, and a stored electron energy of $2.584 \mathrm{GeV}$. This produces a gamma ray beam up to $320 \mathrm{MeV}$ at a rate of about $10^{7}$ photons/sec. In order to completely cover the energy range of the $\Delta$ resonance, it is desirable to increase the energy of the gamma ray beam to greater than $440 \mathrm{MeV}$. This has recently been accomplished by 
two developments: the energy of the electrons in the storage ring was increased to $2.800 \mathrm{GeV}$, and we have increased the laser photon energy by developing the frequency-quadrupled laser to produce 4 watts of $263 \mathrm{~nm}$ light These improvements result in a gamma ray beam energy up to $470 \mathrm{MeV}$ at a rate of about $4 \times 10^{6}$ gammas/sec. The pulse repetition rate of this laser is greater than that of the electron beam $(53 \mathrm{MHz})$, so the time structure of the gamma ray beam is determined only by the electron bunch structure. There are no commercial lasers in existence which meet our requirements. This need has lead us to develop a high power UV ring laser based on the resonant doubling of a high repetition rate Nd:YLF laser followed by a second inter-cavity doubling to produce the UV output.

The use of external resonant cavities can enhance the nonlinear conversion process. It has been recognized that resonating either the fundamental ${ }^{4}$, second harmonic $(\mathrm{SH})^{5}$, or both ${ }^{6}$ can increase the efficiency by more effective conversion of the drive power. Continuous wave $(\mathrm{CW})$ lasers and quasi-CW mode-locked lasers have been doubled to the green with efficiencies of more than $60 \%$ in external resonant cavities. The laser output is stored in an external cavity and allowed to build up to circulating powers orders of magnitude larger than the drive laser output.

Fourth-harmonic radiation has been successfully produced in an all-solid-state system ${ }^{7.9}$. The advantage of this type of system is small size, good power output $(1.5 \mathrm{~W}$ at $266 \mathrm{~nm}$ ), and a low noise all solid-state drive laser. The primary difficulty for lasers in this regime is that the CW IR output cannot be efficiently converted to UV without a high gain cavity in order to achieve large enough power densities for the nonlinear frequency conversion process.

In this approach, a single cavity contains two frequency doubling crystals, one for IR-to-green and one for green-to-UV conversion $^{10}$. The second harmonic is recirculated to resonantly enhance the conversion of the fundamental (pump) radiation and to store a high peak SH power to increase the generation of the fourth-harmonic UV radiation. Since the fourth harmonic of the pump laser is produced directly in the slave cavity, the finesse is a minimum at large output power, making the cavity easier to stabilize.

\section{EXPERIMENTAL DETAIS}

\subsection{Cavity Construction}

The pump laser is a Coherent Corporation Antares model mode-locked Nd:YLF laser which produces $18 \mathrm{~W}$ (CW) output power at $1053 \mathrm{~nm}$. The output pulse frequency is $76 \mathrm{MHz}$ with a pulse width of $50 \mathrm{ps}$ (FWHM) so that the peak output power is about $4.7 \mathrm{KW}(0.24 \mu \mathrm{J})$ per pulse. The IR output of the pump laser is coupled to the slave cavity via a mode matching ("zoom") telescope (TL1 and TL2 in figure 1) which is capable of producing an IR waist from $30 \mu \mathrm{m}$ to $60 \mu \mathrm{m}$ (1/ $\mathrm{e}^{2}$ radius) inside the first doubling crystal (LBO). The IR is steered by two mirrors which are mounted in piezoelectric motor controlled gimbal mounts, (TM1 and TM2), and the beam motions are monitored by two quadrant photodiodes (QPD1 and QPD2). The $\mathrm{x}$ - and $\mathrm{y}$-axis quadrant difference signals are used in feedback loops to stabilize the transverse coordinates of the pump beam. This pointing stabilizer feedback control system has unity gain bandwidth of about $50 \mathrm{~Hz}$ and is phase-lag compensated. The servo maintains the horizontal and vertical beam position to $0.1 \mu \mathrm{m}$ RMS at the LBO crystal.

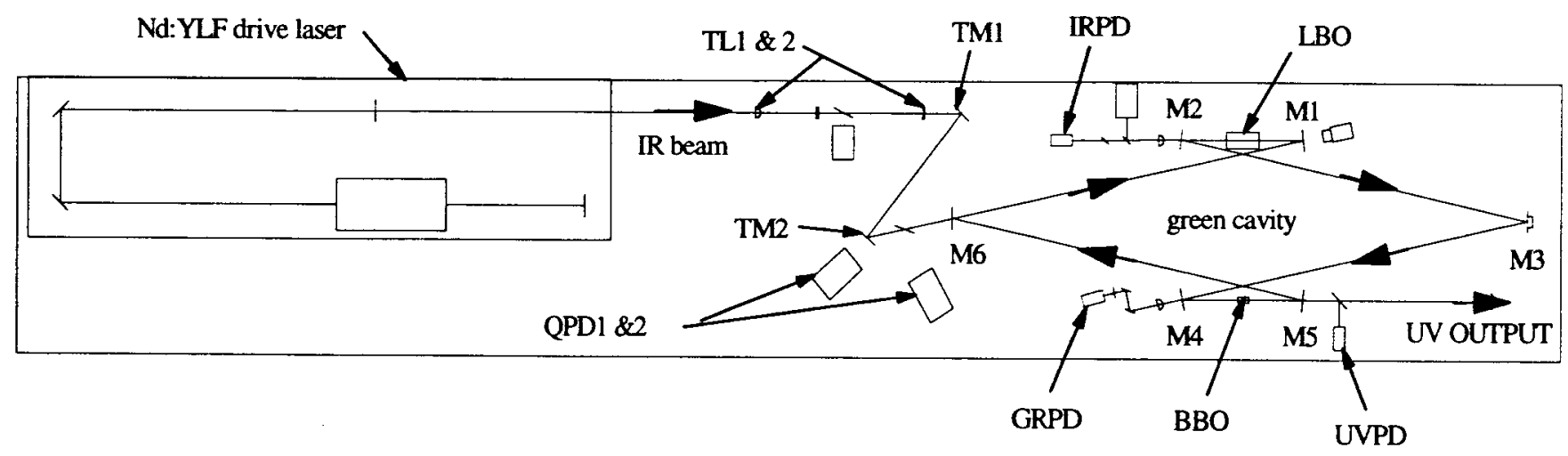

Figure 1. Frequency quadrupling cavity layout on optical bench 
The coherently enhanced ring cavity is comprised of six mirrors (MI through M6) and two non-linear second harmonic generating crystals (SHG). The pump IR enters the ring cavity trough a dichroic input coupling mirror [M6, highly reflecting (HR) at $526 \mathrm{~nm}$, highly transmitting (HT) at $1053 \mathrm{~nm}$ ] and is focused on the first SHG crystal via a $25 \mathrm{~cm}$ radius of curvature (ROC) concave mirror (M1). The first SHG crystal is Lithium Triborate (LBO) which is $15 \mathrm{~mm}$ long crystal cut for type I noncritcal phase matching at $162^{\circ} \mathrm{C}$. The IR exits the cavity from a second $25 \mathrm{~cm}$ ROC concave dichroic mirror (M2, HR at $526 \mathrm{~nm}, \mathrm{HT}$ at $1053 \mathrm{~nm})$. The cavity design is symmetric with the same waist size $(40 \mu \mathrm{m})$ in both the LBO and the second SHG crystal so that mirror pairs M2-M4 and M5-M1 act as 1:1 telescopes for the $526 \mathrm{~nm}$ beam. The second harmonic light is relayed to the second SHG crystal by cavity mirror M3 (HR at $526 \mathrm{~nm}$ ) which is mounted on a piezo-motor for cavity length control. The second SHG crystal is a type I critically phased matched (cut for $48.4^{\circ}$ phase matching angle) $\beta$-Barium Borate (BBO) crystal which converts the $526 \mathrm{~nm}$ beam to $263 \mathrm{~nm}$ light that exits the cavity through the dichroic output coupling mirror (M5). The unconverted $526 \mathrm{~nm}$ light is re-circulated back to the LBO crystal where it is coherently enhanced by the next input IR pulse.

The free spectral range (FSR) of the slave ring matches the mode spacing of the pump laser, so that each longitudinal mode in the pump laser resonates in the coherently enhanced slave cavity. Since the phase sensitivity of the coherent enhancement is directly proportional to the cavity finesse it is necessary to control the cavity length to within a fraction of a wavelength. Presently, the slave ring length is locked to the pump laser by a dither and lock servo system. The piezo-motor mounted mirror $\mathrm{M} 3$ is oscillated at $40 \mathrm{KHz}$ (below its loaded resonance frequency of $43 \mathrm{KHz}$ ), and a photodiode detector monitors the leakage power from cavity mirror M4. The signal is fed to a synchronous detector and used in a Proportional and Integral (PI) feedback loop to control the cavity length. The unity gain crossover for the control system is $900 \mathrm{~Hz}$ and is set by changing the pole frequency of the PI compensator.

\subsection{Cavity length feedback control}

The length of the coherent enhanced cavity must be controlled to a fraction of a wave length for efficient second harmonic conversion to occur. There are numerous sources of noise that are generated by mechanical and optical components in the system and by the surrounding environment. Several methods were employed to measure the relative bandwidth and amplitude of these disturbances on the ring cavity. One method was to use part of the cavity as an interferometer and measure the motions of various optic mounts and system noise. The mechanical resonaces and acoustic noise were measured to be below $500 \mathrm{~Hz}$ with the maximum peak occurring at about $288 \mathrm{~Hz}$ with an amplitude of $54 \mathrm{~nm}$ RMS. This is also evident in accelerometer measurements taken on the optics bench, drive laser head, and mirror mounts. In addition, there is presumably phase noise in the mode-locking, which will effectively appear as cavity length noise. This noise dominates the FSR of the cavity, so that the system is not be stable without an active feedback system to control the cavity length. The bandwidth specifications for the feedback compensator require a unity gain bandwidth of greater than $500 \mathrm{~Hz}$ to control this system.

The power stored in the ring cavity is sensed by a photodetector, which is an integrated photodiode-amplifier package (UDT model $020 \mathrm{UV}$ ) with sensitivity of $55 \mathrm{~W} / \mathrm{V}$ of the power in the cavity (the power detected is the leakage field from cavity mirror M4, with about $0.2 \%$ transmission at $526 \mathrm{~nm}$ ) and a pole frequency of $100 \mathrm{kHz}$. This signal is used for input to a lockin amplifier (Stanford Research Systems model SR510) that has an input frequency response to $100 \mathrm{kHz}$ and a bipolar output with a fixed (a modification to the output amplifier) output pole frequency of $4 \mathrm{kHz}$. This enables the user to provide the dominant pole when used in a feedback loop. This has been realized by the PI feedback module implemented for this system. The feedback module (which is an "in house" design) has a fixed gain integrator (about $43 \mathrm{~dB}$ ) and variable gain proportional leg (range 1 to 20) with adjustable single pole filter (range $50 \mathrm{~Hz}$ to $5 \mathrm{KHz}$ ). Both signals are summed and are fed to another variable gain amplifier with adjustable single pole filter identical to the proportional leg. The error signal is then summed with the modulation signal and is DC offset to be sent to the piezo-motor amplifier (this amplifier is unipolar). The modulation signal is generated by the lock-in amplifier and is $10 \mathrm{mV}$ peak to peak at $40 \mathrm{kHz}$ and is attenuated to $4 \mathrm{mV}$ typically when injected into the feedback module. The piezo-element selected for this system is of stacked design (PI model P-249.10) with a maximum expansion of $5 \mu \mathrm{m}$ at $1000 \mathrm{~V}$. This is driven by a high-voltage amplificr (Trek model 50/750) with a variable gain (voltage gain range from 15 to $300 \mathrm{~V} / \mathrm{V}$ ) and is capable of delivering 0 to $1500 \mathrm{~V}$ with a small signal frequency response of $30 \mathrm{kHz}$. 


\subsection{Monitor detectors}

There are four main detectors used to monitor optical power in this system: the QPD2 sum signal measures input IR, IRPD measures output (depleted) IR, GRPD measures stored green, and UVPD measures output UV. All these detectors are integrated photodiode-amplifier packages (see section 2.2) and were calibrated individually by comparison to a calibrated power meter under steady-state operation of the ring. The calibrated powers can be used to compute a power balance for the ring. The input IR power must equal the IR output plus the UV output plus the green losses in the cavity. The green loss is computed as the fraction of the measured stored green power required to make the power balance sum to zero. An accurate estimate of the fractional power loss can be made by measuring the power balance over a wide range of input IR power and fitting the loss fraction to minimizing the RMS value of the power balance. Cavity power loss estimated in this way agrees well with the decay of the circulating beam observed with a fast photodiode when the cavity length is increased so that consecutive pulses do not overlap. By making many measurements under a wide range of input power, ring conditions and SHG crystals, we find consistently an RMS error in the power balance of $300 \mathrm{~mW}$. This total error must be apportioned between the four detectors in the power balance, so each can be assigned an error of roughly $150 \mathrm{~mW}$.

\section{RESULTS}

The coherent enhanced ring cavity can be characterized by understanding the performance of its four essential elements: the drive laser, the two SHG crystals, and the second harmonic storage ring itself. The characteristics of the drive laser have been discussed above. The measured conversion efficiency of the SHG crystal used for the first doubling (IR to green) and the second doubling (green to UV) are described in Sections 3.1 and 3.2. The evaluation of the ring cavity with only the first SHG crystal present in order to determine the maximum power that can be stored in and extracted from the cavity with the coherent enhancement effect is discussed in Section 3.4. These measurements are compare with simple calculations. The complete ring introduces the second SHG crystal into the cavity to produce UV, and is discussed in Section 3.3 in its present form, and in an improved form in Section 3.5. The UV output is compared to the green power extracted without the second SHG crystal.

\subsection{LBO Single Pass Efficiency}

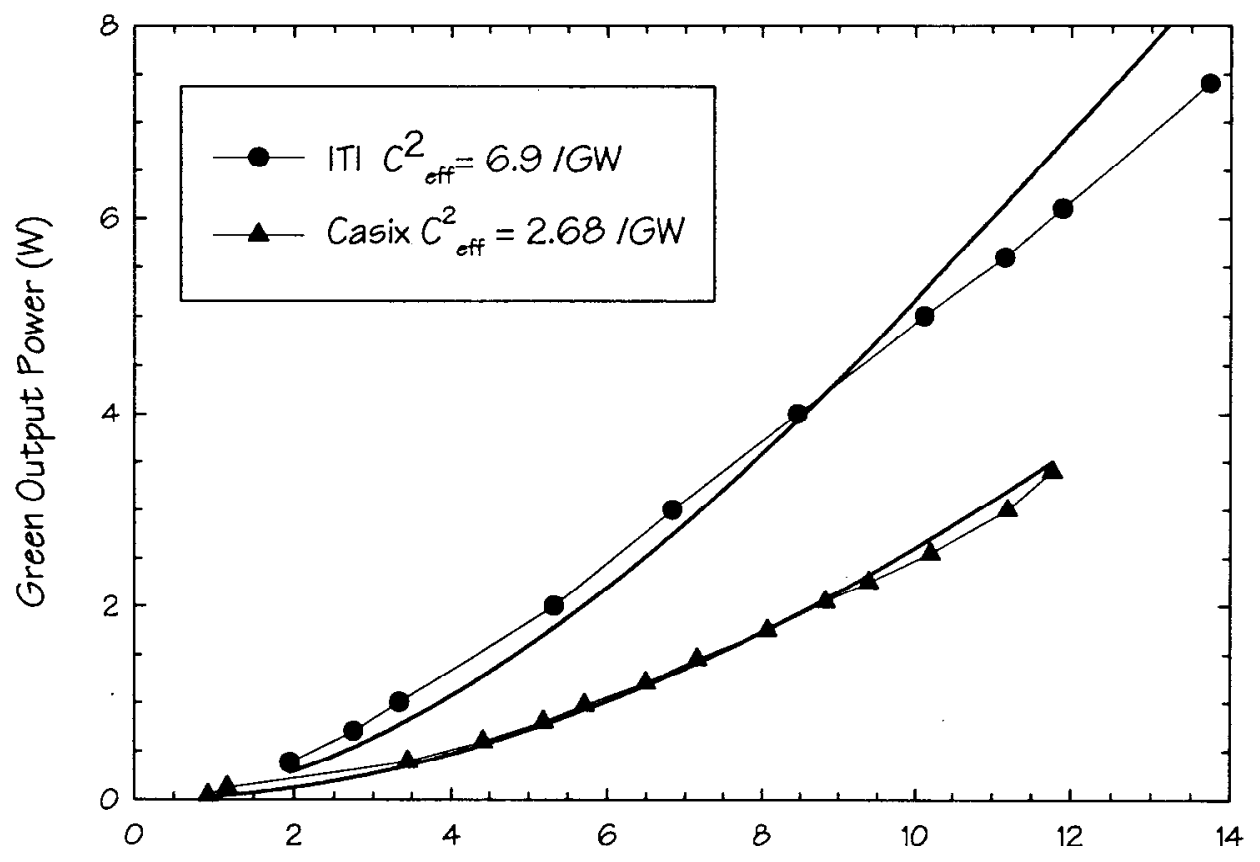

$\mathbb{R}$ Input Power (W)

Figure 2. Single pass output power from LBO 
Two different LBO crystal were used in these studies (both $15 \mathrm{~mm}$ in length), one manufactured by Casix Inc. and the other ITI Electro-Optics Corp. The ring cavity was blocked so that none of the green light produced could recirculate back to the crystal to be enhanced by the next IR pulse (the phrase "single pass" is used to describe this mode of operation). The crystals were mounted in a crystal oven placed at the IR waist between cavity mirrors MI and M2 (see figure 1 ) and the position and temperature was adjusted for optimal green production. As can be observed in figure 2, the SHG efficiency is different between the two manufacturers. The data was fit to the basic equation for SHG

$$
\frac{P_{2 w}}{P_{w}}=\tanh ^{2}\left[\ell \sqrt{c_{e f f}^{2}}\right]
$$

where $\ell$ is the crystal length, $/$ is the fundamental wave power density, and $C_{e f f}^{2}$ is an adjustable parameter "figure of merit"11, including the ponlinear susceptibility as well as dephasing and diffraction effects in the crystal. The deduced values for $C_{\text {eff }}^{2}$ are indicated in Figure 2.

\subsection{BBO Single Pass Efficiency}

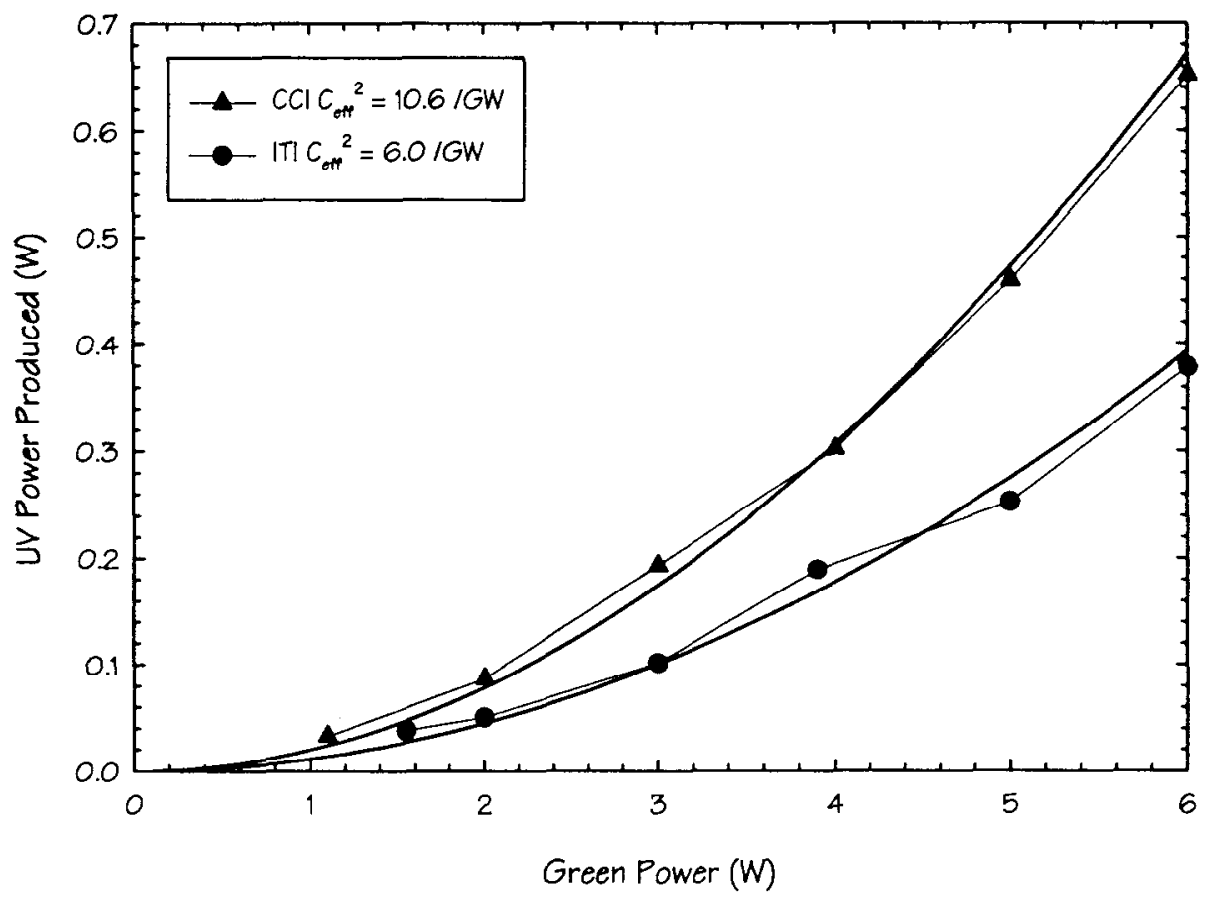

Figure 3. BBO single pass power output

Several different BBO crystals were evaluated using the single pass green produced from the LBO crystal. The BBO crystals were placed at the waist between cavity mirrors M4 and M5 and positioned for optimal UV production. Figure 3 shows the SHG efficiency for two $6 \mathrm{~mm}$ long crystals (one from ITI and the second from Cleveland Crystals Inc.). The effective SHG efficiency is consistently smaller than the $54 / \mathrm{GW}$ quoted in the literature for a plane wave fundamental beam. For critical phase matching (as in BBO) the effect of beam separation due to double refraction (walk-off) can be approximated by reducing the material constant $\mathrm{C}$ according to

$$
\begin{gathered}
c_{e f f}^{2}=\frac{c^{2}}{1+\ell / \ell_{a}}, \text { with } \ell_{a}=\frac{\omega_{e} \sqrt{\pi}}{\rho} \\
\text { and } C=\frac{2.75 d_{e f f}}{\lambda_{S H} \sqrt{\left(n_{o}^{F}\right)^{3}}}
\end{gathered}
$$


Here $\ell$, is the aperture length of the crystal (effective crystal length where harmonic generation can take place), $d_{\text {eff }}$ is the effective nonlinear coefficient (in $\mathrm{pm} / \mathrm{V}$ ), $n_{o}^{F}$ is the ordinary index at the fundamental, and $\rho$ is the walk-off angle of the $S H$ beam. For the design waist size of $40 \mu \mathrm{m}$ at the BBO crystal, the aperture length is $0.8 \mathrm{~mm}$. The expected value of $C_{\text {eff }}^{2}$ for a $6 \mathrm{~mm}$ BBO crystal is $6.4 / \mathrm{GW}$. We observe this value for the ITI crystal; for the CCI crystal we find a value $30 \%$ larger.

\subsection{Production of UV by the Resonant Ring}

The ring cavity for quadrupling IR has been operated for the last six months to produce Compton backscattered gamma rays for nuclear physics experiments. During that time it has produced an average output power of 1.8 watts. The reflection losses in the BBO and output coupler total $18 \%$, so the UV produced averages 2.2 watts. The measured profile of the freely propagating $U V$ beam indicates that it is very close to diffraction limited, with an $M^{2}$ value of 1.3 in the plane of the ring (the walk-off direction) and 1.0 in the vertical plane. However, the walk-off in the relatively long BBO crystal produces a strongly astigmatic beam, with a far field size ratio of $2.5: 1$, so cylindrical optics must be used to transport it to the laser-electron interaction region 22 meters from the exit of the ring. The measured waist size, at the center of the interaction region, is 1000 $\mu \mathrm{m}$ (horizontal) by $500 \mu \mathrm{m}$ (vertical), compared to the diffraction-limited size of $500 \mu \mathrm{m}$ by $500 \mu \mathrm{m}$. The gamma flux produced is $70 \%$ of that expected for a diffraction limited $263 \mathrm{~nm}$ beam, by calculation of the overlap for the two propagating beams. In this calculation we use the measured tunes and emitances of the stored electron beam to integrate the luminosity of the two colliding beams through the six magnetic quadrupole lenses in the $11 \mathrm{~m}$ collision region of the storage ring.

The UV power output of the quadrupling cavity is presently limited by several processes. The first limitation is that the dielectric coatings on the BBO crystal and the UV output coupling mirror (M5) are damaged by the UV. The green antireflection coating on the BBO crystal is Cleveland Crystal Corporation "Solgel". At 3 watts of power in the UV beam (a power density of $16 \mathrm{MW} / \mathrm{cm}^{2}$ ) this coating fails with a mean lifetime of about 2 hours. The resulting reflection loss $(8 \%)$ of green in the cavity reduces the stored power by about $30 \%$, and therefore reduces the UV output to an asymptotic value of about 2 watts after several hours. In addition, the highly reflective dielectric coating on the output coupling mirror is damaged by the $U V$ power (at a density of $0.7 \mathrm{MW} / \mathrm{cm}^{2}$ ) with a mean lifetime of about 5 hours. This damage causes the cavity losses to increase until the UV output is reduced to well below 1 watt, low enough so that the reflective coating no longer degrades. The combined effect of these two damage processes is to reduce the UV output beam to about 1 watt after one day of continuous operation. By translating the BBO crystal and the output coupling mirror a few times per day to expose fresh surfaces to the beam, the UV output power can be maintained at an average value between 1.5 and 2 watts.

The maximum UV output power that can be obtained from the quadrupling ring, during times short compared to the damage times mentioned above, is still limited to 3.4 watts (4.1 watts produced). This is due to the dominance of high order HermiteGaussian modes at high IR pump power. As the pump power is increased above 8 watts (a UV power of 2 watts), the mode in the cavity suddenly switches from relatively pure $\mathrm{TEM}_{00}$ to predominantly $\mathrm{TEM}_{01}$. By placing an aperture in the cavity near one of the focusing mirrors, the dominance of higher order modes can be postponed until 3 watts output, but at the expense of much increased green power loss, and therefore much higher IR input (14 watts). Scanning the cavity length to examine the free spectral range of the cavity reveals many transverse modes. $\mathrm{TEM}_{\mathrm{nm}}$ modes up to $\mathrm{n}+\mathrm{m}=4 \mathrm{can}$ be distinguished. The fraction of the total power in these transverse modes grows with increasing total stored power until the power in one of them (generally $\mathrm{TEM}_{01}$ ) becomes comparable to the $\mathrm{TEM}_{00}$ mode, at which point the cavity length servo locks the length to maximize the higher mode.

The dominance of high order Hermite-Gaussian modes is encouraged by the fact that the present IR transport optics provide an IR beam at the LBO crystal that matches the cavity eigenmode. Since the SHG process produces a green beam with a waist that is smaller than the IR, the green mode is mismatched to the cavity, and higher order modes are introduced. Also, absorption of the UV (and, to a lesser extent, of the green) in the BBO crystal create thermal gradients, and therefore index of refraction gradients. To lowest order these gradients add a power dependent focusing element to the ring, which further mismatches the input mode. Higher order thermal gradients distort the green wavefronts, increasing the power in non-TEM ${ }_{00}$ modes as the UV output increases. In addition, because the UV beam diverges from the green at the walk-off angle $\left(4.9^{\circ}\right)$, the refractive gradient is asymmetric with respect to the green beam. This causes the ring to become misaligned with increasing power, further limiting the maximum power that can be obtained. In spitc of the presence of higher order modes in the ring and of the walk-off of the UV, the output UV beam is nearly diffraction limited. In the vertical (non-walk-off) plane the beam 
propagates as a pure $\mathrm{TEM}_{00}$ mode, indicating negligible contribution of the green non-TEM $\mathrm{D}_{\infty}$ modes to the SHG process. In the horizontal (walk-off) plane, the measured beam phase space is about $1.5 \lambda$ at 2 watts.

\subsection{Performance of the Green Ring}

In order to better understand and optimize the operation of the entire frequency quadrupling system, we have studied the performance of the green storage ring itself: that is, with the BBO crystal replaced by a quartz plate to extract green light. By observing the transient behavior of the cavity, during an FSR scan, thermal effects can be eliminated or at least minimized. The magnitude of thermal phase distortions and misalignments can then be estimated by comparison with steady-state measurements. Such effects are small for the green ring alone.

The stored green power is inversely proportional to the fraction of the internal power that is coupled into the output beam by the green sampling plate. The power extracted from the ring has a maximum value at a coupling that depends on the cavity losses, which are caused by imperfect reflectivity of the mirrors, aperture diffraction, and absorption in the LBO. The computed green output power as a function of the coupling fraction, shown in Figure 4 for 14 watts of input IR, illustrates these features. These calculations ${ }^{5}$ use plane waves and a rectangular time distribution to solve the coupled equations for the fundamental and SH wave interaction in the nonlinear crystal. With no cavity loss the limiting value of output power at a coupling of 1 is just the "single pass" conversion, which is $7.3 \mathrm{~W}$ for the ITI crystal ( $C_{-f}^{2}=6.9 / \mathrm{GW}$ ) and $4.1 \mathrm{~W}$ for the Casix crystal ( $C_{\text {eff }}^{2}=2.7 / \mathrm{GW}$ ). An essential feature of this frequency doubling ring is the coherent enhancement of the IR to green conversion by the presence of the circulating green power in the optical storage ring. The calculation shows this enhancement as the increase in green output as the coupling is reduced from unity and more green power is allowed to circulate back to the LBO to enhance the SHG process. For $4 \%$ cavity losses (the measured value) with the ITI LBO, the maximum power that can be extracted according to the calculation is $10.4 \mathrm{~W}$, an enhancement of $3.1 \mathrm{~W}$, or $42 \%$, over the single pass value. For the Casix LBO, the maximum power is $7.8 \mathrm{~W}$, for an enhancement of $3.7 \mathrm{~W}$, or $93 \%$, over the single pass value. The positive feedback in the enhancement process tends to minimize differences in the single pass efficiency.

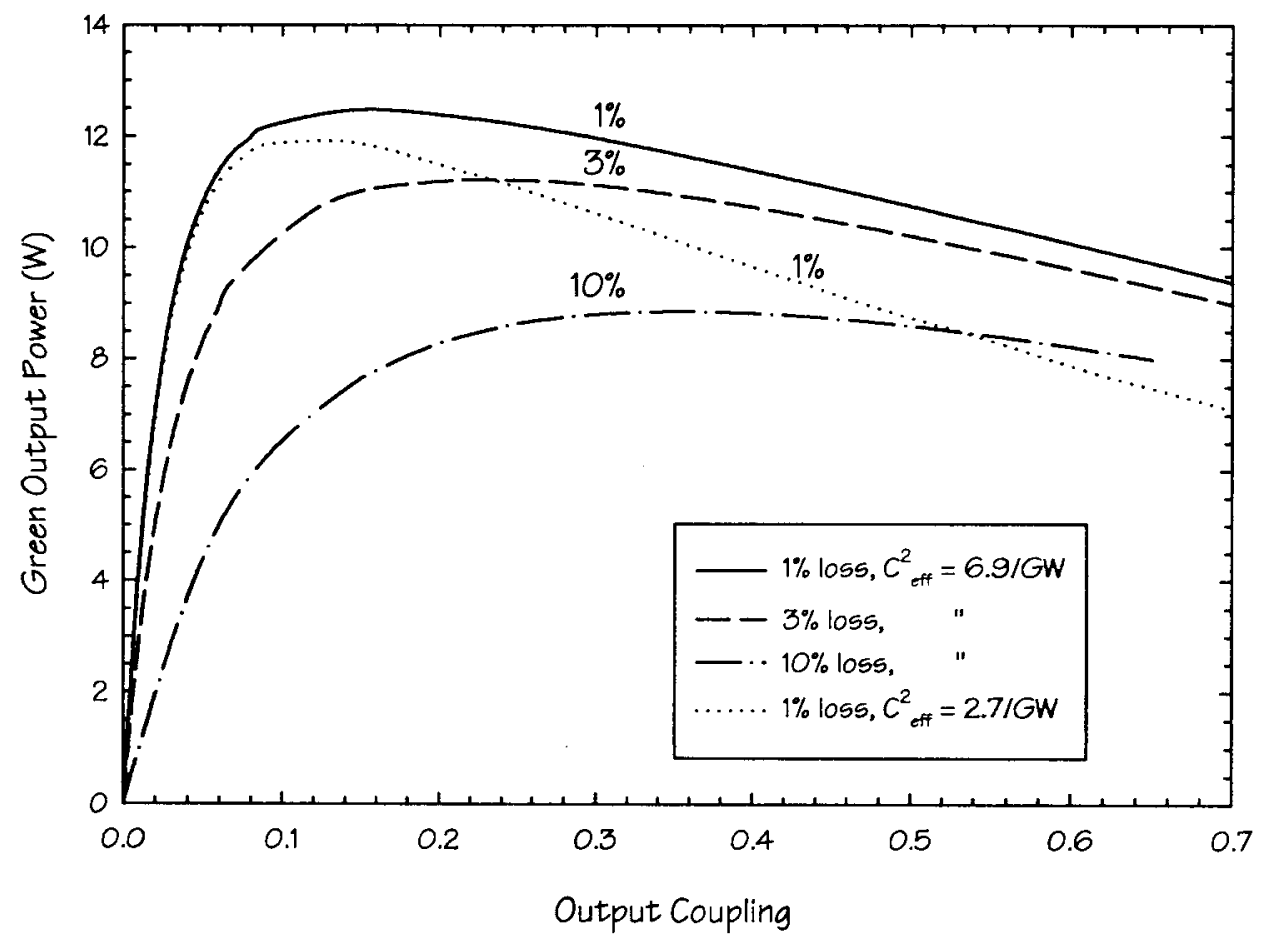

Figure 4. Green output power vs. output coupling for two LBO crystals and for cavity losses of $1 \%, 3 \%$, and $10 \%$ at an IR input of $14 \mathrm{~W}$ 
The enhancement of the conversion process can be measured directly by comparing the depletion of the IR beam (1$\left.P_{\mathbb{R} \text {,our }} / P_{\mathbb{R}, \text { in }}\right)$ with and without re-circulation of the green beam. This is shown in Figure 5 for an output coupling of $11.7 \%$ which produces the maximum extracted green power. The calculated depletion (the dashed line) has roughly the same shape and magnitude as the measured depletion (the solid points) for the ring recirculating green. The solid lines in this figure are smooth curves that fit the data. The open circles are the IR depletion with the ring open (single pass green). The resonant enhancement, measured by the difference between these two data sets, increases the measured depletion by $28 \%$ independent of IR input power above 5 watts. At a pump power of 14 watts, this implies that with resonant enhancement 3.9 watts more SHG green power is delivered to the cavity for conversion to UV, in reasonable agreement with the simple calculation above.

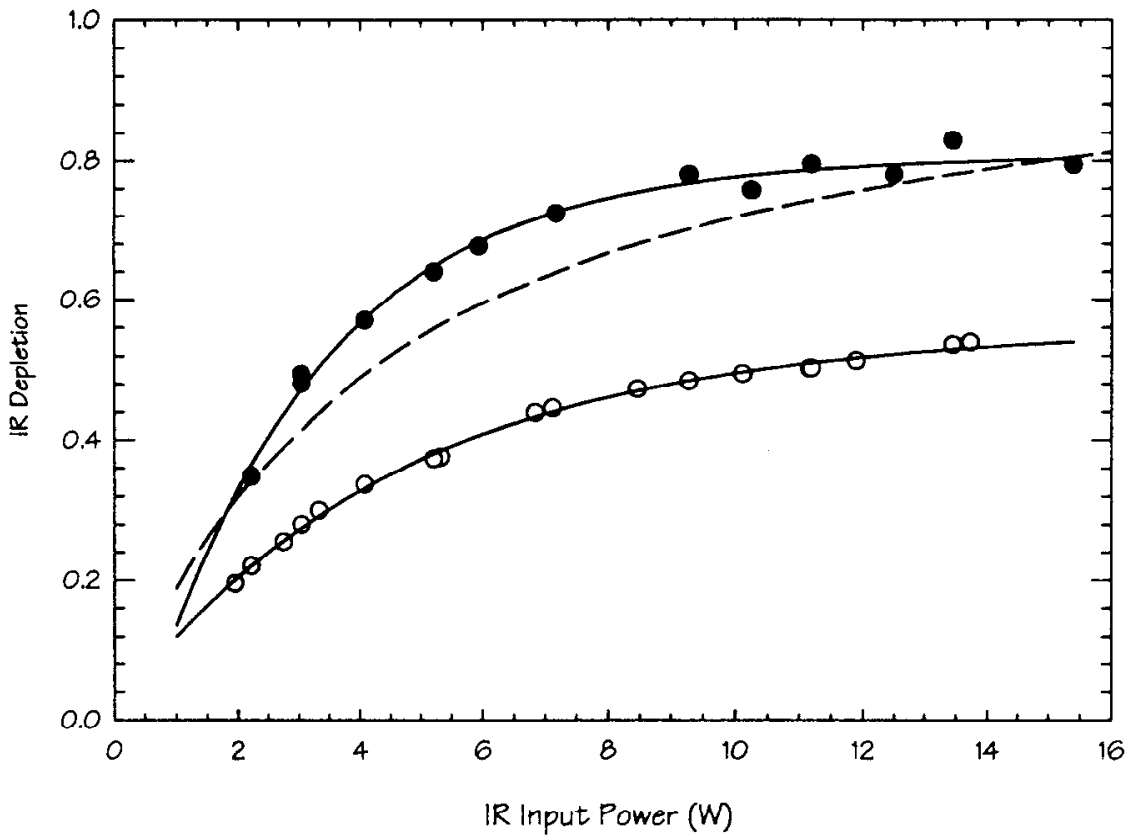

Figure 5. IR Depletion with (solid circles) and without (open circles) resonant enhancement.

The green second harmonic power is generated in the LBO crystal with a waist size that is $1 / \sqrt{2}$ of the waist size of the fundamental IR. The cavity is designed to have an eigenmode which has a waist at the LBO crystal with a size $w_{\text {cav }}(38 \mu \mathrm{m}$ horizontally and $42 \mu \mathrm{m}$ vertically). In order to fill the cavity with its eigenmode the IR beam must have a waist size $w_{I R}$ $=\sqrt{2} \mathrm{w}_{\mathrm{cav}}(54 \mu \mathrm{m}$ by $59 \mu \mathrm{m})$. However, when this is the case, the recirculated green beam at the LBO crystal does not completely overlap the IR beam, and the resonant conversion of IR to green is enhanced less than it would be if the two beams had identical waist sizes. These two conflicting requirements on the mode of the green beam require that either the maximum injected green power or the green mode quality be sacrificed to improve the other. The maximization of IR depletion has been demonstrated by a calculation ${ }^{12}$ to occur when $w_{\mathbb{R}}=w_{\mathrm{cav}}$. Their calculation produces a factor of approximately two reduction in depletion for a factor or two change from this "optimum" IR waist size; no results are shown for the relavent case $w_{\mathbb{R}}=\sqrt{2} w_{c a v}$. However, since the calculation indicates a large increase in IR conversion with the IR beam waist size matched to the cavity mode, we have always operated the quadrupling ring in this condition.

Because, as discussed above, the presence of higher order modes in the stored green beam limit the UV output to 3.4 watts, it is important to determine how much the depletion actually decreases with the proper mode matching condition. To do this we have used the zoom telescope, described in Section 2.1, to vary the IR to a waist from 30 to $60 \mu \mathrm{m}$ at the LBO crystal. The depletion, and the beam modes, have been measured as a function of the IR input beam waist size.

The transverse modes stored in the cavity are shown in Figure 6 when either the IR beam or the green beam is matched to the cavity eigenmode. As expected, when the green beam is matched to the cavity eigenmode $\left(w_{\mathbb{R}}=57 \mu \mathrm{m}\right)$ the cavity is filled with an essentially pure $\mathrm{TEM}_{00}$ mode (the heavy line in Figure 6). Only a very small amount of $\mathrm{TEM}_{01}$ mode is also present, 
and that is only because the modes of both the cavity and the input IR beam are astigmatic and the matching is correct only in the horizontal plane. In contrast, when the IR is matched to the cavity ( $w_{\mathbb{R}}=40 \mu \mathrm{m}$ ) higher order modes are strongly populated in the cavity. In particular, the TEM $\mathrm{TE}_{01}$ and TEM 10 modes can be produced with $30 \%$ of the power of the TEM mode. The location of these transverse modes, marked in the figure, agrees well with the eigenvalues for the ABCD matrix computed from the measured ring geometry. There obviously is a strong preference for matching the green beam to the cavity to avoid producing higher order modes.

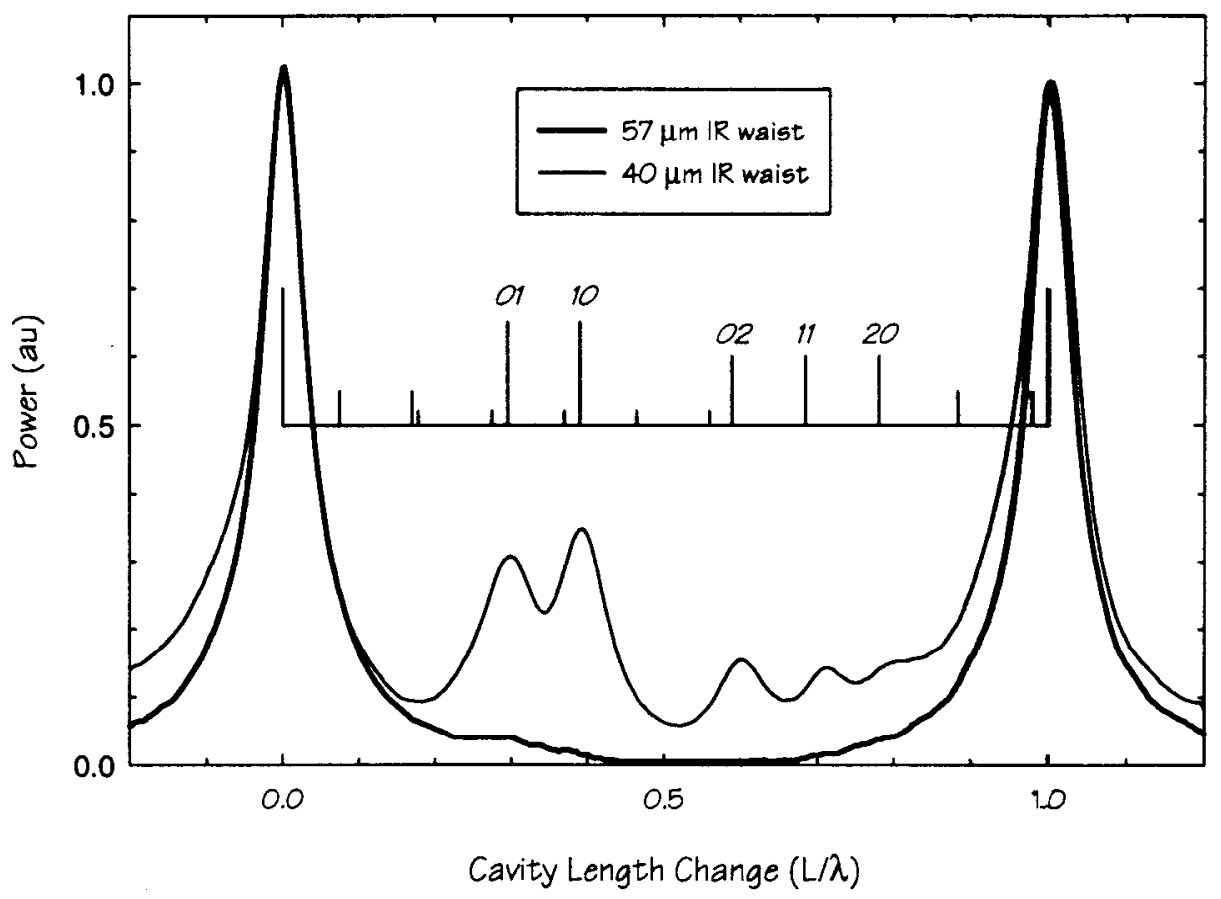

Figure 6. Transverse modes for an IR waist at the LBO to match green to cavity $(57 \mu \mathrm{m})$ and to maximize depletion $(40 \mu \mathrm{m})$

To investigate the effect of the mode matching conditions on the efficiency for converting to green, we have measured the depletion and the extracted green power with the sampling plate extracting $11.7 \%$ of the stored green power. The results are shown in Figure 7. The open squares are the measurements for an IR waist size of $57 \mu \mathrm{m}$ at the LBO crystal. The solid line is a fit of an exponentially rising function to the data, for reference. The dash-dot line is the same function fitted to the data for an IR waist of $40 \mu \mathrm{m}$, which produces the maximum depletion (this is the data of Figure 5). The depletion with the green mode matched to the cavity is only 7\% lower than the maximal value. At 14 watts IR input power, this leads to 1 watt extra green power input to the ring. Clearly, the penalty in conversion efficiency for property matching the green mode to the cavity is much smaller than the calculation ${ }^{12}$ indicates.

In fact, the measurements show no advantage in matching the IR mode to the cavity: the stored green power is measured to be identical for the two mode matching conditions. The data for the green output power with the green matched to the cavity mode $\left(w_{\mathbb{R}}=\mathbf{5 7} \mu \mathrm{m}\right)$ is shown by the circles in Figure 7. The solid circles are for the "transient" condition, measured as the cavity length is swept through the resonance condition, and the open circles are for the "steady-state" condition with the cavity length servo locked to the resonance condition. The heavy solid line is the green output power calculated with the model described above. The thin solid and dashed lines are respectively the linear regression lines for the transient and steady-state conditions. The slightly lower output power for the steady state condition is presumably due to small thermal distortions in the LBO crystal, causing either a mode shift due to thermal lensing or a cavity misalignment. The regression lines for the green output with the IR waist matched to the cavity mode are indistinguishable from those shown in the figure. This occurs because with the IR matched more higher order modes are produced, which have higher diffraction losses on the small cavity length control mirror. The measured cavity losses for the "matched IR" condition are 4.8\%, whereas for the "matched green" condition they are 3.6\%. This difference can also be seen in Figure 6 as the increased width (lower finesse) 
for the "matched IR" condition. The extra $1.2 \%$ cavity loss, at a stored power of 70 watts, accounts for an extra 0.8 watts of green lost in the ring, which just cancels the 1 watt gained by the higher IR depletion.

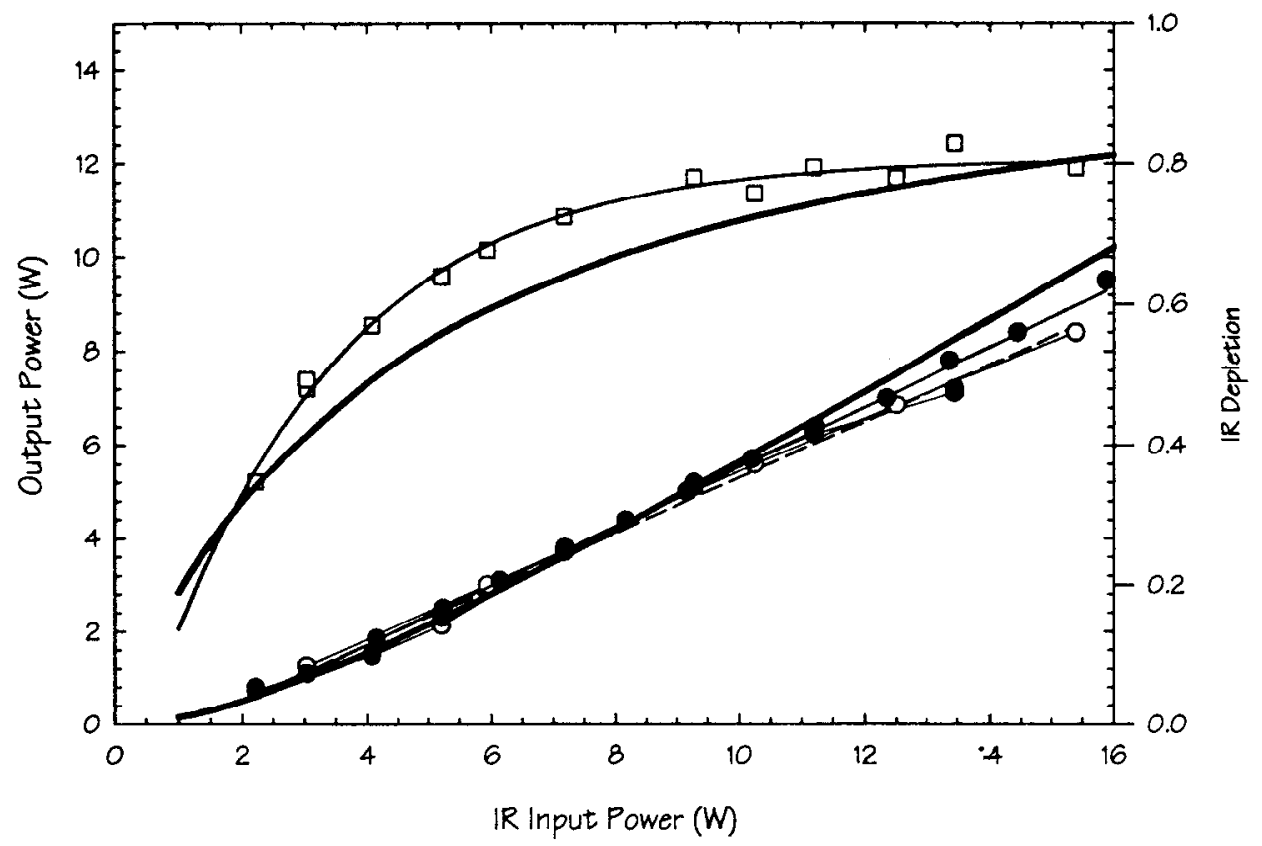

Figure 7. Extracted green power (circles) and IR depletion (squares) with $11.7 \%$ sampling plate and $57 \mu \mathrm{m} \mathrm{IR}$ waist at LBO

\subsection{Performance to be Achieved by an Improved Ring}

We are currently modifying the frequency quadrupling ring to correct the deficiencies we have discussed above. The first step is to remove some dielectric coatings from the path of the UV beam. To avoid the damage to the green antireflection coating on the BBO crystal we have replaced the present rectangular crystal with one which has faces cut at the Brewster angle for $526 \mathrm{~nm}$ (the ITI crystal in Figure 3). To eliminate damage of the UV output coupling mirror, M5, we are redesigning the cavity to include a dual-AR coated prism after the BBO crystal, which will separate the two beams enough that the UV can miss M5. A dual-AR coating may be more "robust" to UV damage than the dichroic coating of the present M5. The reflection loss for the UV in the Brewster cut crystal is $22 \%$, which is similar to the UV losses in the present ring with undamaged BBO and output coupler ( $18 \%$ ). We expect the UV loss for a good dual-AR coating to be less than $0.5 \%$, so that the total losses for the new scheme should be $23 \%$. However the green reflection - and the UV output - losses will always remain at the low undamaged values.

The performance of the ring with the Brewster cut crystal and the "matched green" IR input mode in the present green ring with the existing undamaged output coupler is shown in Figure 8. The open squares and circles are the data for IR depletion and UV output, respectively. The solid circles are the steady-state UV output data. Dashed lines are best fit lines to the data. This data can be compared to the depletion and green power output power for the ring with the BBO crystal replaced by the $11.7 \%$ sampling plate. To make this comparison meaningful for the power output, the green power must be reduced by $30 \%$ to mimic the UV reflection losses in the BBO (22\%) and M5 (10\%). The solid lines are the IR depletion and "corrected" green output, taken from Figure 7.

The measured UV output is 4.25 watts at 14 watts IR pump power. This corresponds to 5.9 watts of UV produced in the BBO at a measured IR depletion of $69 \%$ ( 9.7 watts of green into the ring), with 102 watts of green stored, and 3.6 watts of green lost. When the cavity is modified to allow the UV to miss the output coupler, the output power will increase to 4.7 watts. 


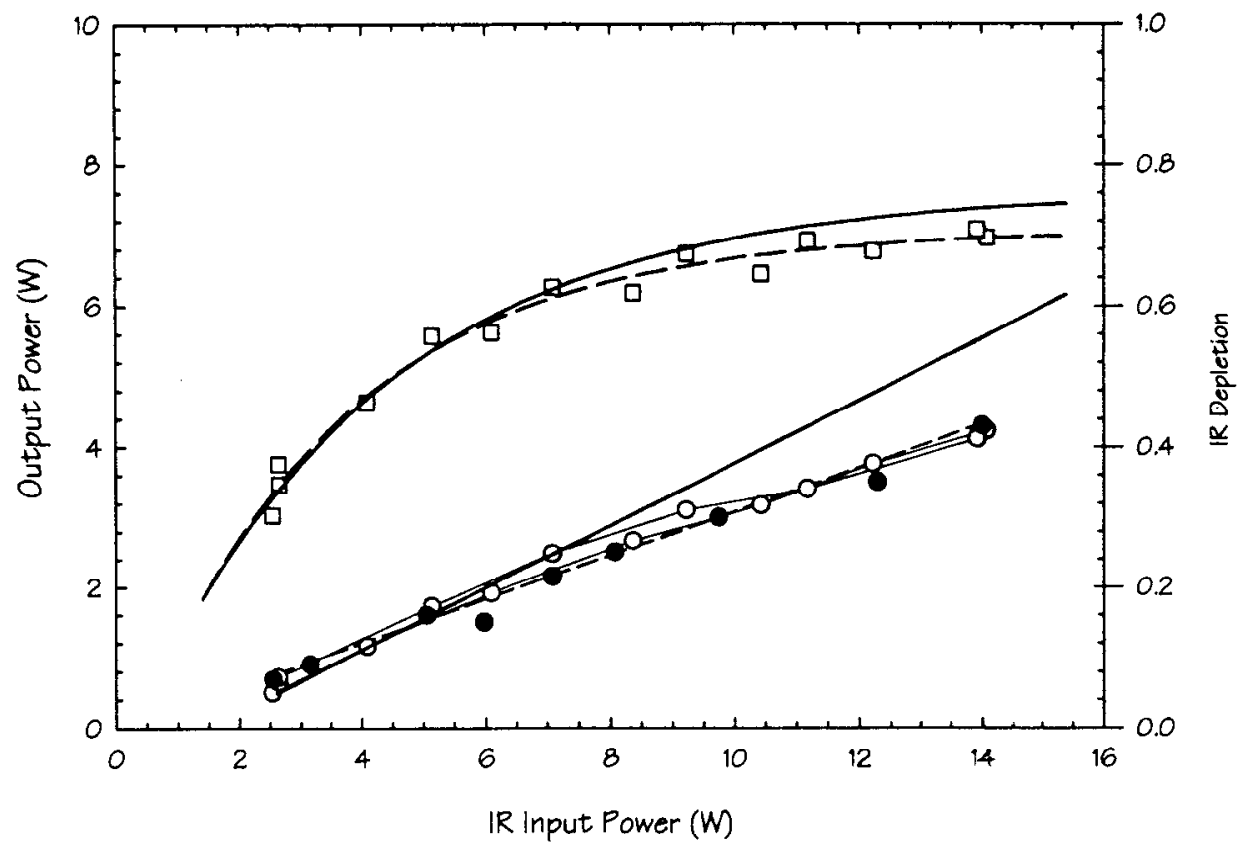

Figure 8. UV power output (circles) and IR depletion (squares) with $6 \mathrm{~mm}$ Brewster cut BBO

At high pump power, the UV output power output falls well below that would be expected for a constant $12 \%$ extraction of power from the green ring (the solid line). This is explained in part by increased ring losses with the BBO present (5.1\% compared to $3.6 \%$ with no BBO). Equally important, at the high green power stored in the ring, the efficiency of the BBO $(6 \%$ at $100 \mathrm{~W})$ is much lower than expected from the single pass measurements (11\% at $6 \mathrm{~W})$. This reduced output coupling results in a lower extracted power for the UV than for the green, as is expected from the calculations shown in Figure 4 . We believe that the decrease in SHG efficiency of the $\mathrm{BBO}$ at high power is caused by thermally induced misalignments of the cavity by the $\mathrm{BBO}$ crystal, which increase the size of the stored green beam with increasing power.

\section{CONCLUSION}

We have constructed and are presently operating a ring for frequency quadrupling 14 watts of IR to produce up to 3.4 watts of useable UV power. The UV beam has been transported 22 meters to a nearly diffraction limited waist of $1 \mathrm{~mm}$ by $0.5 \mathrm{~mm}$. The UV beam phase space is less than 2 times the diffraction limit up to the maximum power, and since it is dominated by walk-off in the BBO crystal, it varies only weakly with power. This beam has been used continuously for 2 weeks to produce a gamma ray beam by Compton scattering from an electron beam stored in a synchrotron. Recent improvements in the ring have increased the UV output to 4.2 watts, and further developments, in progress, can reasonable be expected to yield up to 5 watts.

The resonantly enhanced green ring performance can be modeled quite well by a simple calculation. Matching the mode of the SH green to the cavity mode, rather than the input IR as has previously been done, results in a much more nearly pure $\mathrm{TEM}_{00}$ cavity mode with no significant reduction in stored green power. We have extracted 9.5 watts of green power from the ring with 15.9 watts of IR input, for an output conversion efficiency of $60 \%$. The low conversion efficiency of the BBO crystals at high green power presently limits the output UV power. 


\section{ACKNOWLEDGMENTS}

We want to thank Brian Perkins for writing a Gaussian optics program, Luca Matone for help with the measurements, Frank Lincoln for fabricating mechanical components, and Enio Turi for designing and constructing electronics for the control system. This work has been supported by the U.S. Department of Energy under Contract No. DE-AC02-76CH00016 and by the Instituto Nazionale di Fisica Nucleare.

\section{REFERENCES}

1. A.M. Sandorfi, M. Levine, C.E. Thom, G. Giordano, G. Matone, and C. Schaerf, "High Energy Gamma Ray Beams from Compton Backscattered Laser Light", I.E.E.E. Trans. Nucl. Sci. NS-30, 3083 (1983).

2. G. Blanpied, et.al., "N $\mathrm{N} \rightarrow \Delta$ Transition from Simultaneous Measurements of $\mathrm{p}(\gamma, \pi)$ and $\mathrm{p}(\gamma, \gamma)$ ”, Phy. Rev. Lett., Vol. 79, No. 22, pp 4337-4340, (1997).

3. J. Tonnison, A.M. Sandorfi, S. Hoblit, and A.M. Nathan, "First Extraction of a Spin Polarizability of the Proton", Phy. Rev. Lett., Vol. 80, No. 20, pp 4382-4385, (1998).

4. S. T. Yang, et. al., "6.5 W, $532 \mathrm{~nm}$ Radiation by CW Resonant External Cavity Second Harmonic Generation of an 18 W Nd:YAG Laser in $\mathrm{LiB}_{3} \mathrm{O}_{5}$ ”, Opt. Lett., Vol. 16, pp. 1493-1485, (1991).

5. G.Giordano and G.Matone, "A Ring-Cavity for a Quadrupled Nd:YAG Laser", Il Nuovo Cimento, Vol. 12, pp. 927-933, (1990).

6. A. Askin, G.D. Boyd and J.M. Dziedzic, "Resonant optical second harmonic generation and mixing", IEEE J. Quantum Electronics, QE2, 109 (1966).

7. M.Oka, et.al., "All Solid-State Contiuous-Wave Frequency-Quadrupled Nd:YAG Laser", IEEE J. Quantum Electronics, Vol. 1, No. 3, pp. 859-865, (1995).

8. A.S. Bell, G.P. Malcolm and G.T. Maker, "High Power Continuous Wave 266nm Source", SPIE Proceedings, Vol. 3613 , No. 13. (1999).

9. E. Zanger, et.al., "Diode-Pumped High-Power CW all Solid-State Laser at 266nm", SPIÉ proceedings, Vol. 3613, No. 45, (1999).

10. S.C. Tidwell, et.al., "Efficient high-power UV generation by use of resonant ring driven by a CW mode-locked IR laser", Opt. Lett., Vol. 18, pp. 1517-1519. (1993).

11. D. Eimerl, "High Power Harmonic Generation", IEEE J. Quantum Electronics, Vol. QE-23, No. 5; pp 575-592, (1987).

12. M.S. Bowers, S.C. Tidwell, J.F. Seamens and D.D. Lowenthal, "Diffractive Analysis of an External Resonant Ring for Quadrupling Nd Lasers", OSA Proceedings, Vol. 15, pp131-133,(1993). 